\title{
Study on Emergency Evacuation and Intervention Behavior of Population with Spatial Knowledge Integrity
}

\author{
Xuan Wang \\ Sichuan Normal University \\ Yan Mao ( $\sim$ maoyy85@163.com ) \\ Sichuan Normal University \\ Jing Jing Xiong \\ Sichuan Normal University \\ Wu He \\ Sichuan Normal University
}

\section{Research Article}

Keywords: behavior, emergency

Posted Date: December 3rd, 2021

DOI: https://doi.org/10.21203/rs.3.rs-1109930/v1

License: (c) (i) This work is licensed under a Creative Commons Attribution 4.0 International License. Read Full License 


\section{Abstract}

The level of spatial knowledge integrity of a population is crucial for fire escape behavior. The use of appropriate interventions for people with different levels of spatial knowledge can effectively improve evacuation efficiency. However, different emergency situations also have different effects on evacuation behavior. In this paper, we combine spatial knowledge integrity, intervention behavior and emergency situations in a fire evacuation study. To complete this study, 128 participants were recruited using VR technology, classified into spatial knowledge completeness, and studied crowd evacuation through different intervention behaviors in different simulated emergency scenarios. The results of the study showed that participants with complete spatial knowledge had shorter evacuation distances and times. Secondly, leader interventions guided evacuation better for participants with incomplete spatial knowledge in low-hazard emergencies, while range interventions were better for participants with complete spatial knowledge. Thirdly, in high-risk emergencies, leader intervention was better than range intervention for evacuation, regardless of spatial knowledge completeness. Fourth, the interaction between spatial knowledge completeness and intervention behavior was significant, positively influencing the evacuation time and distance of participants.

\section{Introduction}

Fire is a great calamity. For example, the 1115 fire in Shanghai killed 58 people ${ }^{11}$. Due to the rapid progress of urbanization, rapid development of commercial economy, the scale of a single building is constantly expanding, and the internal structure is becoming more and more complex. To improve the efficiency of evacuation and reduce casualties, the problem of evacuation in emergency has become a research hotpot.

The efficiency of evacuation in emergency depends on the completeness of spatial knowledge ${ }^{19}$. Having good spatial knowledge helps to reduce evacuation time and distance ${ }^{23,35}$.The influence of spatial knowledge integrity on evacuation is mainly reflected in the following aspects: first, the evacuation efficiency of participants with different spatial knowledge integrity is different. Participants with complete spatial knowledge were able to find evacuation routes more quickly ${ }^{23}$. The participants with incomplete spatial knowledge cannot identify the environment, which leads to the difficulty of evacuation ${ }^{35}$.Therefore, participants with complete spatial knowledge are more likely to reach the safe area than participants with incomplete spatial knowledge ${ }^{16}$.Secondly, people with different spatial knowledge have different evacuation characteristics, such as: people with complete spatial knowledge rely on autonomous evacuation ${ }^{43}$.People with incomplete spatial knowledge were evacuated with the surrounding crowd ${ }^{19}$.Thirdly, people with different levels of spatial knowledge tend to choose familiar evacuation routes ${ }^{36}$.People in emergency situations tend to choose familiar routes and exits rather than unfamiliar routes ${ }^{1,34}$. However, depending only on the spatial knowledge integrity of individuals to choose evacuation routes may lead to crowd congestion ${ }^{46}$, outlet blockage ${ }^{5}$, evacuation efficiency reduced ${ }^{1,34}$ and issues et al. Therefore, some scholars in the study of spatial knowledge based on the 
integrity of leaders, signs and crowd flow patterns and other factors to solve the existing problems in crowd evacuation ${ }^{19,23}$. However, the impact of spatial knowledge integrity on population evacuation under different dangerous emergency degrees is unclear.

Effective intervention can improve the efficiency of crowd evacuation. Intervention refers to the behavior that the leader himself or the leader provides the security information to the crowd in the emergency situation through the equipment ${ }^{42,46}$. There are two kinds of intervention: leader intervention and scope intervention ${ }^{42,46}$. Leader intervention is the behavior of trained leaders directly entering the site to provide evacuation information and guide people from dangerous areas ${ }^{24,27}$.Scope intervention refers to the act of providing people with security information such as routes and exits through broadcasting ${ }^{21}$ and marking ${ }^{24}$.The scenarios for leader intervention are as follows: first, people cannot accurately access environmental information. Because the vision of the people is limited by the smoke or the lack of illumination, the leaders' intervention becomes the main guiding behavior ${ }^{7}$. Secondly, the information acquired by personnel is uncertain. When a fire occurs in the tunnel, the warning information obtained by the personnel through the FM radio is uncertain, which leads to the invalidation of the scope intervention guidance $^{4}$. The scope of intervention is as follows: first, the disabled escape. Disabled people with visual impairment are sensitive to LED signs in fires and can be effectively identified for escape ${ }^{3}$. Secondly, the route or exit is blocked. In the event of a fire or blockage at the exit, a dynamic identification system will display a warning message, which will be recognized by the evacuee, who will change the direction of movement and find a new evacuation route ${ }^{9}$. Thirdly, there are not enough leaders. In large public places, such as stadiums, due to the lack of leaders, people mainly by identifying signs to evacuate ${ }^{23}$. Although the effect of intervention behavior on evacuation in emergency has been studied, the effect of intervention behavior on evacuation in different dangerous emergency degree is not clear.

The degree of spatial knowledge integrity is an important factor affecting intervention behavior. For people with incomplete knowledge of space: first, the leader intervenes. Leaders directly into the site to conduct emotional evacuation and provide evacuation routes ${ }^{42}$, the experiment proved to be effective in guiding the evacuation of personnel ${ }^{5,10}$. But the number of leaders should not be too many, generally $10 \%$ of the total number of people for the best ${ }^{26,27}$. Second, scope intervention. The leader intervenes all the individuals in the space by means of information diffusion, such as broadcasting, and asks them to evacuate in order according to the signs ${ }^{42}$.For example, in large public places, the crowd for the environment is mostly unfamiliar, in case of emergency mainly follow the signs to evacuate ${ }^{23}$. For those with a complete knowledge of space: first, the intervention can enhance their familiarity with the route. Be familiar with route blocking and be able to navigate other routes ${ }^{27,46}$.Secondly, when the fire occurs, the smoke will affect people's line of sight, in different visibility, light or sound can effectively guide the evacuation ${ }^{37}$.Finally, with the development of intelligence, the intelligent guiding evacuation system and signs developed for crowd evacuation also have good results ${ }^{2}$. However, the effect of the interaction of spatial knowledge integrity and intervention behavior on crowd evacuation is still unclear. 
The degree of urgency of different risks is another important factor influencing intervention. The evacuation behavior is different in different dangerous emergency degree, such as: In Low Dangerous Emergency, people tend to scatter to escap $\mathrm{e}^{44}$.In high-risk emergencies, people tend to congregate, often following others to escape, causing crowds, blocked exits and inefficient evacuations ${ }^{7}$. The intervention behavior is different for different emergency degree. For example, in low risk emergencies, fire information is provided to people through smoke detector and sound alarm systems to improve evacuation efficiency ${ }^{6,8}$.In high-risk emergencies, the ability to move people is significantly reduced, and leaders can more effectively provide evacuation guidance and reduce casualty rates ${ }^{6,8}$. However, in real life, the participants' spatial knowledge integrity, intervention behavior and the degree of danger and urgency are integrated into one, for example, subway, shopping mall fire in the environment of people with different spatial knowledge integrity ${ }^{19,32}$, studying the intervention behavior of this emergency has realistic significance.

To test the behavior of crowd evacuation in fire emergency, there are four methods in the previous research: First, computer simulation. For example, by building a model to simulate the dynamic behavior of crowd evacuation, study the efficiency of crowd evacuation ${ }^{15,25,29}$. Or include influencing factors to the underlying model, such as the effect of leaders on population evacuation ${ }^{36}$,Effect of emotional contagion on crowd evacuation ${ }^{22}$.Second, the questionnaire survey. Investigate crowd behavior and characteristics, as well as crowd self-organization phenomena during evacuation, such as route and exit choice ${ }^{14}$, herd behavior ${ }^{30}$ et al. Third, the simulation experiment ${ }^{2,15}$.Fourth, the virtual evacuation experimental study ${ }^{38,39}$. However, these methods have some limitations, such as lack of real population data or incomplete data, high cost, difficult to set up a controlled experimental environment, and so on. So virtual reality

(VR)-based immersive virtual environments (Ives) offer an alternative to crowd evacuation studies. Virtual reality is "A real or simulated environment in which the perceiver experiences telepathy" ${ }^{37}$. The evacuation behavior observed in virtual reality environments is qualitatively comparable to real-world behavior ${ }^{17}$.

IVE is an effective research tool with reasonable ecological validity, which can arouse human emergency behavior. It can control and manipulate key variables according to experimental design, and collect reliable qualitative and quantitative behavior data ${ }^{18,47}$.In this paper, the method based on IVE is used to solve three problems in this study. First, what is the effect of spatial knowledge integrity on evacuation behavior in virtual fire scenarios? Second, what effect does the degree of spatial knowledge integrity have on evacuation behavior under different emergency levels? Third, what effect does the interaction of spatial knowledge integrity and intervention behavior have on evacuation behavior under different emergency levels?

\section{Methods}

\section{Methods}




\section{Participants}

The participants in this study were 128 students or social participants from Sichuan Normal University, China (average age $=20.2$, ranging from 18 to 22 years; 64 men and 64 women). They had normal or corrected to normal vision (Visual acuity above 1.0) and made no difference in color identification (noncolor-blind color). Participants were required to sign an informed consent form prior to the start of the experiment. Following this, participants were asked to complete the pre-experiment questionnaire, including questions about participant basic information, positive effects, and negative impact PANAS scale ${ }^{40}$. Each subject was paid 50 CNY. The study was approved by the Ethics Committee of Sichuan Normal University. All experimental procedures were carried out in accordance with the Declaration of Helsinki.

\section{Instruments}

This experiment was carried out at the Sichuan Normal University. We used the VR system, and the integrated resolution of the binocular display was $(1080$ (horizontal) $\times 1200$ (vertical)) pixels. We used 3D Studio Max software to model and render IVE (immersive virtual environments) and then import it into the platform of the Unity3D gaming engine. The Unity3D particle system was also used to visualize the spread of fire and smoke in cases of fire emergency experiments, using headphones to provide emergency broadcasting and fire sound.

During the experiment, participants maintained a standing position, interacting with the IVE using the operating lever. Specifically, they manipulate the lever to move in the IVE at a constant speed of $1.2 \mathrm{~m} / \mathrm{s}$, moving forward, backward, left, or right at their own speed while keeping the body still.

\section{Virtual display}

This study used the flat floor of a virtual large shopping mall displaying a variety of different commercial shops. The mall is 50 meters long, 40 meters wide and 4 meters high. As can be seen from the scene diagram of Figure 1, there are two entrances, and two emergency exits in the mall. In this large virtual shopping mall, there are 16 shops, all of which are in an open state. Furthermore, participants were not allowed to cross the walls of the room.

As shown in Figure 1, there is a promotion point symbol (marked by red police flag), with a fire point is the promotion point, as an experiment of fire emergency. This paper simulates two experimental scenarios, each where fire and smoke spread across the $3 \mathrm{~m} \times 4 \mathrm{~m}$ area of the ground, which can be seen from anywhere in the mall (fire and smoke in Figure 2)

\section{Experimental process}

The study adopted the 2(spatial knowledge integrity: complete spatial knowledge, incomplete spatial knowledge) X 2(risk emergency: low emergency, high emergency) X 2(intervention behavior: leader intervention, scope intervention) model among the participants. In this experiment, subjects were divided 
into two large groups with equal numbers and equal male to female proportions, each divided into four more groups, respectively A, B, C, D, E, F, G, H. The first group was asked to complete two tasks namely promotion and evacuation, and the second group only required evacuation. The promotion point task is to be familiar with the mall environment along the specified route (Figure 3 ) and needs to find the correct one from 6 maps, consisting of 5 interference maps and one correct map, formed on the correct map by adding or deleting some building elements (e. g., outlet and store location) or changing the location of existing building elements. The evacuation mission is an emergency escape mission at the promotion point, (there will be 1 minute of adaptation time before performing the mission). The screen appears: "The fire occurs, please evacuate as soon as possible". Evacuation mission began and participants performed emergency evacuation by selecting different evacuation routes. (Figure 4)

The experiment was conducted in low-risk and high-risk emergencies, with the low-risk emergency in the low fire and smoke environment with $70 \%$ visibility in the smoke scenario and the fire and smoke environment, with $10 \%$ visibility in the smoke scenario (fire and smoke in Figure 2 ). In addition, crowd evacuation is guided through leader intervention or scope intervention. Leader intervention refers to the leader directly entering the mall to provide emotional comfort and evacuation guide to participants. Scope intervention is to broadcast reminder at each route from the fire point to the exit "do not panic, orderly evacuation according to the evacuation sign".

\section{Experimental validation}

The experimental process is shown in Figure 5. The figure shows that upon arrival at the laboratory, participants were required to sign a consent form before starting the experiment (Figure 3).Following this, participants were asked to complete the pre-experiment questionnaire, including questions about participant basic information, positive effects, and negative impact PANAS scale ${ }^{40}$. Next, participants were asked to read the instruction manual on how to use IVE navigation in the system. They then need to perform the training tasks. They will wear HMD, immersion in an empty open space to simply demonstrate IVE, to be familiar with the operation of VR devices and immersion in the virtual environment.

Next, participants in the first group were asked to understand instructions for the promotional point task and then perform the promotional point task as instructed (Figure 1).Participants were not told whether the map they chose correct after the end of the promotional point task, however, only participants who chose the correct map were considered the evacuation outcome valid.

All participants were then asked to perform evacuation missions at the virtual mall and the fire required escape to the exit as soon as possible. After reading the instructions for the evacuation task, subjects needed to perform the evacuation task in an immersive environment. (There will be a minute of adaptation before the mission), the screen appears: "The fire occurs, please evacuate as soon as possible". The evacuation mission began, and virtual fires and smoke emerged. 
Table 1

Evacuation routes

\begin{tabular}{|lllllll|}
\hline Group & Route 1 & Route 2 & Route 3 & Route 4 & Route5 & total \\
\hline A & 0 & 16 & 0 & 0 & 0 & 16 \\
\hline B & 5 & 3 & 2 & 4 & 2 & 16 \\
\hline C & 0 & 16 & 0 & 0 & 0 & 16 \\
\hline D & 3 & 6 & 2 & 3 & 2 & 16 \\
\hline E & 0 & 16 & 0 & 0 & 0 & 16 \\
\hline F & 2 & 2 & 3 & 5 & 4 & 16 \\
\hline G & 0 & 16 & 0 & 0 & 0 & 16 \\
\hline H & 6 & 2 & 4 & 3 & 1 & 16 \\
\hline total & 16 & 77 & 11 & 15 & 9 & 128 \\
\hline
\end{tabular}

There will be different emergencies and interventions for different groups. Two A, B groups in the A, B, C, D of the first group would be placed in a low-hazardous emergency and two C, D groups in a highly hazardous emergency. For A for leader intervention, B for scope intervention, $C$ for leader intervention and $D$ for scope intervention. For the two $E, F$ groups in the $E, F, G, H$ of the second group that were placed in a low hazardous emergency, the two G, H groups were placed in a highly hazardous emergency. For $E$ for leader intervention, $\mathrm{F}$ for scope intervention, $\mathrm{G}$ for leader intervention and $\mathrm{H}$ for scope intervention.

At the end of the experiment, participants were asked to fill out the post-experiment questionnaire PANAS ${ }^{40}$. In addition, the following questions should be answered (5 points): the vividness of the test scene, the walking difficulty of the planned route, and the vertigo.

\section{Results}

Route selection (using one-way ANOVA)

(Table 1) The evacuation route choice of participants in different scenarios was summarized, and the results were analyzed by one-way ANOVA. And it turns out, both the intervention behavior $(\mathrm{F}=21.847$, $P=7.472 E-06<0.05)$ and the degree of danger emergency $(F=4.105, P=0.0450<0.05)$ significantly influenced the participants' choice of evacuation route, the impact on evacuation routes is independent, and the impact of interventions on route selection is greater than that of hazardous emergencies. However, spatial knowledge integrity $(F=2.170, P=0.143>0.05)$ did not significantly affect the participants' route choice, which is not in line with our conventional cognition, and the study of its influencing factors has practical significance. 
Route selection in Table 1 shows that participants with incomplete spatial knowledge have more evacuation route choices than participants with complete spatial knowledge; different intervention behaviors in the fire can also affect participants to choose different routes for evacuation.

\section{Completeness of spatial knowledge}

In this study, we assessed participant evacuation performance by evacuation distance $(\mathrm{m})$ and evacuation time (seconds). The mean values of the two dependent variables are shown in Figure 6.This paper on the evacuation distance and time of evacuation task 2(spatial knowledge integrity: complete spatial knowledge, incomplete spatial knowledge) X 2(dangerous emergency: low emergency, high emergency) $X 2$ (intervention behavior: leader intervention, scope intervention) experiment. The results show that the degree of spatial knowledge integrity has significant effect on evacuation distance $(F=7.924, p=0.006<0.05)$. Participants with complete spatial knowledge had shorter evacuation distance than those with incomplete spatial knowledge( $35.1 \mathrm{~m}$ vs $39.8 \mathrm{~m})$. The results also reveal that the degree of spatial knowledge integrity has a significant effect on evacuation time, $F=3.842, p=0.005<0.05$, This indicates that

the evacuation time of participants with complete spatial knowledge is shorter than that of participants with incomplete spatial knowledge (47.9s vs $55.5 \mathrm{~s})$.

\section{Intervention behavior}

In Figure 7, This study shows that different interventions have a significant impact on the evacuation distance $(F=104.568, p=3.575 E-18<0.05)$. The results showed that participants in the leader intervention had shorter evacuation distance than those in the scope intervention $\nabla 30 \mathrm{~m}$ vs $43.3 \mathrm{~m}$ ). The different intervention behavior also has the remarkable influence on the evacuation time, $F=72.922, p=4.077 \mathrm{E}-$ $14<0.05$, this indicated that the participants' emergency evacuation time under leader intervention was shorter than that under scope intervention (35.9s VS 64s)

\section{Spatial knowledge integrity and intervention in behavioral interactions}

The results show that the interaction of spatial knowledge integrity and intervention behavior has a significant effect on evacuation time $(F=30.027, P=2.277 \mathrm{E}-7<0.05)$ and evacuation distance $(F=5.230, P=0.024<0.050)$, in which the participants can escape more quickly and improve the evacuation efficiency.

\section{Emergency degree}

This paper also conducts for emergency evacuation under different danger emergency 2(spatial knowledge integrity: spatial knowledge, spatial knowledge) X 2(dangerous emergency: low emergency, high emergency) $X 2$ (intervention behavior: leader intervention, scope intervention) experiment. The results show that the degree of danger emergency has no significant effect on evacuation distance $(F=2.721, p=0.102>0.05)$ 
Results were shown in Figure 8, participants in low-risk emergencies had longer evacuation distances than those in high-risk emergencies(38.9 $\mathrm{m}$ vs $36.1 \mathrm{~m})$. The degree of danger emergency also has a significant effect on the evacuation time, $F=14.408, p=0.0002<0.05$, This suggests that participants in a low-risk emergency were evacuated for longer than those in a high-risk emergency(59s vs $44.5 \mathrm{~s}$ ).

\section{Subjective evaluation}

Two participants in the study failed to select the correct sketch and their data were excluded from the results. The following analysis considered a total of 128 participants. Independent sample tests of potential confounding factors showed no significant differences among groups.

\section{Discussion}

\section{Influence of the completeness of spatial knowledge on fire emergency evacuation}

In summary, the results of this study show that the participants' evacuation route choice is determined by the degree of spatial knowledge integrity. Specifically, participants in an emergency evacuation tend to take familiar routes, and participants with complete spatial knowledge are more likely to seek out and choose the best routes ${ }^{11,35}$.(Table 1 Evacuation routes) The vast majority of participants (77) with complete spatial knowledge were evacuated via the shortest route (Line 2). Because participants with complete spatial knowledge understand the whole environment, they can use analytical decision systems to make informed path-finding decisions ${ }^{21,33}$.For the participants with incomplete spatial knowledge, the choice of evacuation route is more random and unpredictable than the participants with complete spatial knowledge $^{35}$ (Table 1 Evacuation routes). Participants with complete spatial knowledge are therefore able to make optimal decisions and find the best routes to reduce hazards when they occur.

In addition, the evacuation efficiency of participants is also determined by the degree of spatial knowledge integrity. The results show (Figure 6) that the participants with incomplete spatial knowledge had longer evacuation distance and time in the evacuation task. Under the condition of incomplete spatial knowledge, the evacuation time (55.5s) and the evacuation distance $(39.8 \mathrm{~m})$ were longer than the participants' evacuation time $(47.9 \mathrm{~s})$ and the evacuation distance $(35.1 \mathrm{~m})$.Under the condition of incomplete spatial knowledge, the participants need more time to perceive the environment and hesitate to make the route decision, which makes the escape more difficult ${ }^{35}$.And having complete spatial

knowledge reduces hesitation, reduces difficulty, and makes evacuation easier to complete ${ }^{12,35}$.So people need to pay more attention to the surrounding information in daily life, remember the significant markers, to achieve the space and self-integration state.

\section{Effect of the intervention behavior on the fire emergency evacuation}


According to the above results, the leader intervention is more effective for crowd evacuation. Figure 7 shows that Participants in the leader intervention condition were evacuated for shorter distances (30 m vs $43.3 \mathrm{~m}$ ) and time (35.9s vs 64s) than participants in the range intervention condition. Because leaders were able to directly enter the venue to guide participants, making security information spread more efficiently ${ }^{24}$.Under the scope of intervention, participants are slow to accept information, and participants need to take the initiative to seek environmental information, resulting in uncertainty in the decisions made, low evacuation efficiency and increased mortality.

Regardless of the intervention behavior, there were implications for participants' escape route selection (Table 1 Evacuation routes). Leaders who are familiar with the environment will give participants environmental information and lead participants to evacuate from the shortest route ${ }^{24}$. Thus, participants guided by leader intervention behavior all chose to evacuate from the shortest route. However, when participants are guided by range intervention, participants need to actively perceive safety information such as identification, before the direction and speed of movement change dynamically 45 .

\section{Interaction effect of spatial knowledge and completeness of intervention behavior}

The interaction of the degree of spatial knowledge completeness with the intervention behavior had a significant effect on the evacuation time and distance of the participants. The findings show that for participants with complete spatial knowledge, leader intervention behavior can pacify participants and reduce evacuation time and distance. and alternative route guidance when familiar routes are blocked ${ }^{27}$. Scope intervention can again determine the correctness of evacuation routes by identifying and broadcasting, enhancing participants' familiarity with routes and reducing evacuation errors ${ }^{24}$.

For participants with incomplete spatial knowledge, the effect of leader intervention behaviors was more effective on evacuation than scope intervention. Leaders can directly lead participants to evacuate from the shortest route, reducing the time for participants to find escape routes and improve evacuation efficiency. Scope intervention allowed participants to find stable and reliable guidance information, such

as emergency export instructions ${ }^{24,41}$. However, since participants do not know environmental information and require longer searching for identification, participants may take longer to find the right route, so participants relied more on leader instructions for evacuation ${ }^{13}$, so scope interventions are less efficient than the leader intervention in evacuation.

\section{Effect of dangerous emergency degree on fire emergency evacuation}

This study demonstrates that participants in highly dangerous emergencies are more likely to escape. The evacuation time and distance of participants in low hazardous emergencies were greater than the time and distance of population evacuation in highly hazardous emergencies (59s vs $44.5 \mathrm{~s} 38.9 \mathrm{~m}$ vs $36.1 \mathrm{~m})$. It was previously proposed that crowds panic in highly dangerous emergencies and that 
pedestrian mobility will be significantly reduced ${ }^{45}$.However, this study proved that under high emergencies, population evacuation is more efficient and has shorter evacuation time and distance, which is inconsistent with previous studies.

In a low-degree dangerous emergency, the crowd can evacuate more calmly because it is not greatly affected, and the highly dangerous emergency will strain the crowd and lead to accelerated action, so the evacuation time is greater than the time of people to walk in a highly dangerous emergency ${ }^{45}(59 \mathrm{~s}$ vs 44.5s).

Evacuation distance decreases in high hazard emergencies compared to low hazard emergencies $(38.9 \mathrm{~m}$ VS $36.1 \mathrm{~m}$ ). Because of highly dangerous emergencies, participants form cluster states that favor collective escape ${ }^{7}$.In low-risk emergencies, participants tended to escape with increased randomness of route selection ${ }^{44}$, resulting in reduced population evacuation efficiency.

\section{The three interactions}

In conclusion, the interactive effects of spatial knowledge integrity, intervention behavior, and different emergencies have a significant impact on population evacuation. In low-risk emergencies, for participants with incomplete spatial knowledge, leader intervention provides better guided population evacuation (31.7s vs $74.2 \mathrm{~s} 30 \mathrm{~m}$ vs $45.8 \mathrm{~m}$ ) relative to scope intervention. Participants with incomplete spatial knowledge needed more time to find escape routes due to a lack of knowledge of environmental information. Although labeling was important for participants to find escape routes, leaders were able to guide evacuation more efficiently ${ }^{10}$.

For participants with complete spatial knowledge, scope intervention provides better guided evacuation behavior (44.6s vs 61.9 s $30 \mathrm{~m}$ vs $41.2 \mathrm{~m}$ ) relative to leader intervention. Participants with complete spatial knowledge tend to choose "safe" routes that they know, rather than seeking additional environmental cues, trying alternative routes that they are unfamiliar with ${ }^{19}$.So although leader intervention can lead participants to evacuate from the best path, people are still more likely to confirm their correct decisions by identifying familiar routes.

In highly dangerous emergencies, leader intervention performs better evacuation guidance than scope intervention (32.5s VS $56.8 \mathrm{~s} 30 \mathrm{~m}$ VS $42.3 \mathrm{~m}$ ), whether spatial knowledge is complete. In highly dangerous emergencies, panic reached a maximum, decreased perceptual function and increased loss of orientation, resulting in difficult evacuation decision-making and preventing the population to escape as soon as possible ${ }^{27}$.And the smoke can interfere with the population identification of evacuation signs ${ }^{27}$. But trained leaders can remain emotionally calm in highly dangerous emergencies and make correct decisions rationally to guide effective evacuations.

\section{Conclusions And Limitations}


The results show that: first, the distance and time of participants with complete spatial knowledge are shorter in emergency evacuation. Second, in a low-risk emergency, leader intervention is better for participants with incomplete spatial knowledge to guide evacuation; participants with complete spatial knowledge are better for scope intervention. Third, in highly dangerous emergencies, leader intervention evacuation better than scope intervention whether spatial knowledge is complete. Fourth, the interaction between the completeness of spatial knowledge and the intervention mode was significant, with a positive effect on the evacuation time and distance of the participants.

A key advantage of our study is using VR technology for virtual reality simulation. Previous studies rely on computer simulations and often fail to realistically reflect real population evacuation behavior. The technique is applied in the experiment to realistic the evacuation behavior, and the research results are more realistic.

Another advantage of our study is the application of different emergency scenarios to experiments. Fire changed dynamically, but previous studies conducted only one simulation of an emergency scenario, and the observed population evacuation behavior was not comprehensive enough. Therefore, the study of different emergency scenarios can be more objective and accurate analyzed and enhance the practical significance of experiments. This paper shows that population evacuation efficiency is higher in high emergencies than in low emergencies, overturning the results of previous studies.

The results of this study have three practical implications, firstly, shopping malls have complete spatial knowledge. In emergencies, they strongly tend to follow the familiar route for evacuation, so their evacuation route choices are highly predictable. This means that the mall emergency department can investigate the composition of the personnel, predict the route of emergency evacuation according to the spatial knowledge of the personnel, and formulate emergency plans accordingly. Secondly, participants with incomplete spatial knowledge tend to move slower and stochastic road finding decision-making, suggesting that they are a major challenge for emergency evacuation. These personnel lack knowledge of the environment and rely heavily on environmental cues to make their own way-finding decisions. Therefore, during the emergency evacuation, guidance information should be displayed through signs and staff instructions to help people with safe evacuation. Thirdly, in highly dangerous emergencies, population decision uncertainty increases due to the complex environment and the high-risk factor. The emergency department of the mall should send more leaders to intervene and directly enter the mall to lead the evacuation. In low-risk emergencies, the population tends to escape randomly, targeting participants with complete spatial knowledge, the identification can better ensure that participants walk the correct route. Leaders' participants with incomplete spatial knowledge, better guided evacuation due to a lack of environmental information.

Admittedly, this study also has three limitations that can be addressed in future studies. First, the limitations of intervention behavior, there are many types such as static identification, dynamic identification, trained leaders, mobile devices, mobile robots, and wireless sensor networks ${ }^{46}$. This paper considers only two major intervention behaviors, the trained leaders, and assistive devices. In addition, 
this paper only applies the intervention behaviors separately, and does not consider the impact of using at the same time two or more intervention behaviors on the fire evacuation simultaneously. Secondly, reallife fire is a process from small to large dynamic change. This paper only low-degree fire and heavy fire scenarios are considered, and the scene of fire dynamic change process has not been considered. Whether the results obtained in this paper are applicable to emergency evacuation under dynamic change fire scenarios remains to be studied. Thirdly, this paper only considers the degree of spatial knowledge, but there are people with complete spatial knowledge in real life. It remains to be verified whether the results of this study apply to those with complete spatial knowledge. How people can improve the completeness of their spatial knowledge through spatial exploration, and the impact of the improved spatial knowledge completeness on evacuation behavior is another question worth studying.

\section{References}

1. Anthony, R. Mawson. Understanding Mass Panic and Other Collective Responses to Threat and Disaster. Routledge, 68, 2 (2014).

2. Bernardini, G., Azzolini, M., D’Orazio., M. \& Quagliarini, E. Intelligent evacuation guidance systems for improving fire safety of italian-style historical theatres without altering their architectural characteristics. Journal of Cultural Heritage. 22(Complete), S1296207416301030(2016)

3. Boyce, K. E., Shields, T. J. \& Silcock, G. Toward the characterization of building occupancies for fire safety engineering: capability of people with disabilities to read and locate exit signs. Fire Technol, 35 (1), 79-86 (1999).

4. Caroly, S., Kouabenan, D. R. \& Gandit, M. Analysis of danger management by highway users confronted with a tunnel fire. Safety Science. 60(Complete), 35-46(2013)

5. Casareale, C., Bernardini, G., Bartolucci, A., Marincioni, F. \& D'Orazio, M. Cruise ships like buildings: wayfinding solutions to improve emergency evacuation. Build. Simul, 10 (3), 1-15 (2017).

6. Cc, A., Pc, B., Rdr, A., Mgm, C. \& Pr, D. Modelling and numerical simulation of pedestrian flow evacuation from a multi-storey historical building in the event of fire applying safety engineering tools. Journal of Cultural Heritage, 41, 188-199 (2020).

7. Ding, N. \& Sun, C. Experimental study of leader-and-follower behaviours during emergency evacuation. Fire Saf. J, 117 (1), 103189 (2020).

8. Fang, Z. M., Song, W. G., Hao, W. \& Zhang, J. A multi-grid model for evacuation coupling with the effects of fire products. Fire Technol, 48 (1), 91-104 (2012).

9. Galea, E. R., Xie, H., Deere, S., Cooney, D. \& Filippidis, L. Evaluating the effectiveness of an improved active dynamic signage system using full scale evacuation trials. Fire Safety Journal, 91(jul.), 908917 (2017).

10. Gershon, R., Qureshi, K. A., Rubin, M. S. \& Raveis, V. H. Factors associated with high-rise evacuation: qualitative results from the world trade center evacuation study. Prehosp Disaster Med, 22 (3), 165173 (2007). 
11. ., Guo Xiaohe \& Zhengqin, L. Investigation of "12.25" major fire accident and cause of death in Luoyang Dongdu Commercial Mall.China Fire Association (China Fire Protection Association): China Fire Association, 3(2003)

12. Hoffman, J. E. \& Nelson, B. Spatial selectivity in visual search. Percept. Psychophys, 30 (3), 283-290 (1981).

13. Hou, L., Liu, J. G., Pan, X. \& Wang, B. H. A social force evacuation model with the leadership effect. Physica A Statistical Mechanics \& Its Applications, 400, 93-99 (2014).

14. Huang Hai-Jun. Static floor field and exit choice for pedestrian evacuation in rooms with internal obstacles and multiple exits. Physical Review E Statistical Nonlinear \& Soft Matter Physics, 78 (2), 021131 (2008).

15. Jihyun Kang. In-Jae Jeong., Joon-Bum Kwun. Optimal facility-final exit assignment algorithm for building complex evacuation. Comput. Ind. Eng, 85, 169-176 (2015).

16. Kim Ju Yeon., Choi Jin Kyung., Han Won Hee., Kim Jong Ha. The Influence of Users' Spatial Familiarity on Their Emotional Perception of Space and Wayfinding Movement Patterns. Sensors. 21(8), 2583-2583 (2021)

17. Kinateder, M., Comunale, B. \& Warren, W. H. Exit choice in an emergency evacuation scenario is influenced by exit familiarity and neighbor behavior. Saf. Sci, 106, 170-175 (2018).

18. Kinateder, M., Ronchi, E., Nilsson, D. \& Kobes, M. A Mülberger. Virtual Reality for Fire Evacuation Research. Computer Science \& Information Systems. IEEE. 313-321(2014)

19. Lin, J., Cao, L. \& Li, N. How the completeness of spatial knowledge influences the evacuation behavior of passengers in metro stations: a vr-based experimental study. Automation in Construction, 113, 103136 (2020).

20. Ling, C. M. \& Law, K. H. Incorporating individual behavior, knowledge, and roles in simulating evacuation. Fire Technol, 55, 437-464 (2019).

21. Ando, M. O. T. Tunnelling and Underground Space Technology. underground transportation systems, 90, 28-41 (2019).

22. Mao, Y., Fan, X., Fan, Z. \& He, W. Modeling group structures with emotion in crowd evacuation. IEEE Access, 99, 1-1 (2019).

23. Mei Ling Chu, Parigi, P. \& Law, K. Jean-Claude Latombe. Modeling social behaviors in an evacuation simulator. Computer Animation and Virtual Worlds, 25, 3-4 (2014).

24. Min, Z., Dong, H., Zhao, Y., Zhang, Y. \& loannou, P. Optimal number and location planning of evacuation leader in subway stations. IFAC-PapersOnLine, 51 (9), 410-415 (2018).

25. Mirahadi, F. \& Mccabe, B. Y. Evacusafe: a real-time model for building evacuation based on dijkstra's algorithm. Journal of Building Engineering. 101687(2020)

26. Mccormack, P. \& Chen, T. Optimizing leader proportion and behavior for evacuating buildings. Society for Computer Simulation International, 13, 1-6 (2014). 
27. Pelechano, N. \& Badler, N. I. Modeling crowd and trained leader behavior during building evacuation. IEEE Computer Graphics and Applications, 26 (6), 80-86 (2006).

28. Qiu, L., Zheng, X. \& Wang, Y. F. Revision of the Positive Emotional Negative Emotion Scale (PANAS). Appl. Psychol, 14 (03), 249-254 (2008).

29. Rassia, S. T. \& Siettos, C. I. Escape dynamics in office buildings: using molecular dynamics to quantify the impact of certain aspects of human behavior during emergency evacuation. Environmental Modeling \& Assessment, 15 (5), 411-418 (2010).

30. Ruggiero Lovreglio., Achille Fonzone., Luigi dell' Olio., Dino Borri. A study of herding behaviour in exit choice during emergencies based on random utility theory. Safety Science. 82, 421-431(2016)

31. Seike, M., Kawabata, N. \& Hasegawa, M. Walking speed in completely darkened full-scale tunnel experiments. Tunnelling and Underground Space Technology, 106, 103621 (2020).

32. Seong-Hwan Yoon., Min-Jung Lee., Jurng-Jae Yee. An experimental study on evacuation times in a subway station using evacuation parameters.Journal of Asian Architecture \& Building Engineering. 12(1),93-100(2013)

33. Siegel, A. W. \& White, S. H. The development of spatial representations of large-scale environments. Advances in Child Development \& Behavior, 10, 9-55 (1975).

34. Sime, J. Movement toward the familiar. Environment \& Behavior, 17 (6), 697-724 (1985).

35. Song, D., Park, H., Bang, C., Agnew, R. \& Charter, V. Spatial familiarity and exit route selection in emergency egress. Fire Technol, 55, 2269-2287 (2019).

36. Spartalis, E., Georgoudas, I. G. \& Sirakoulis, G. C. CA Crowd Modeling for a Retirement House Evacuation with Guidance. International Conference on Cellular Automata. Springer International Publishing. 481-491(2014)

37. Steuer, J. Defining virtual reality: dimensions determining telepresence. Journal of Communication, 42 (4), 73-93 (2010).

38. Wang, B., Li, H., Yacine, R. \& Alex, B. Ong, H. N. Bim based virtual environment for fire emergency evacuation.Scientific world journal. 589016(2014)

39. Wang, X., Zheng, X. \& Yuan, C. Evacuation assistants: an extended model for determining effective locations and optimal numbers. Physica A Statistical Mechanics \& Its Applications, 391 (6), 22452260 (2012).

40. Watson, D., Clark, L. A. \& Tellegen, A. Development and validation of brief measures of positive and negative affect: the panas scales. J Pers Soc Psychol, 54 (6), 1063-1070 (1988).

41. Xie, H., Filippidis, L., Galea, E. R., Blackshields., D. \& Lawrence, P. J. Experimental analysis of the effectiveness of emergency signage and its implementation in evacuation simulation. Fire and Materials, 36, 5-6 (2012).

42. Xie, K., Song., Y. \& Liang, B. Panic contagion and intervention strategy in crowd evacuation: from the perspective of complexity science management. Chinese Journal of Management, 16 (02), 273-279 (2019). 
43. Yang, L. I., Chen, J., Zhang, Q. \& Yang, H. University, N. P. Study of pedestrian evacuation model considering familiarity with environment. China Safety Science Journal, 26 (04), 168-174 (2016).

44. Yang, X., Dong, H. \& Yao, X. Passenger distribution modelling at the subway platform based on ant colony optimization algorithm. Simulation Modelling Practice and Theory, 77, 228-244 (2017).

45. Yuan, Z. \& Jia, Hongfei, Z. A social force evacuation model considering the effect of emergency signs. Simulation: Journal of the Society for Computer Simulation, 94 (8), 723-737 (2018).

46. Zhou, M., Dong, H., loannou, P. A., Zhao, Y. \& Wang, F. Y. Guided crowd evacuation: approaches and challenges. IEEE/CAA Journal of Automatica Sinica, 6 (05), 1081-1094 (2019).

47. Zou, H., Li, N. \& Cao, L. J. Emotional Response-Based Approach for Assessing the Sense of Presence of Subjects in Virtual Building Evacuation Studies. Journal of Computing in Civil Engineering. 31,5(2017)

\section{Figures}

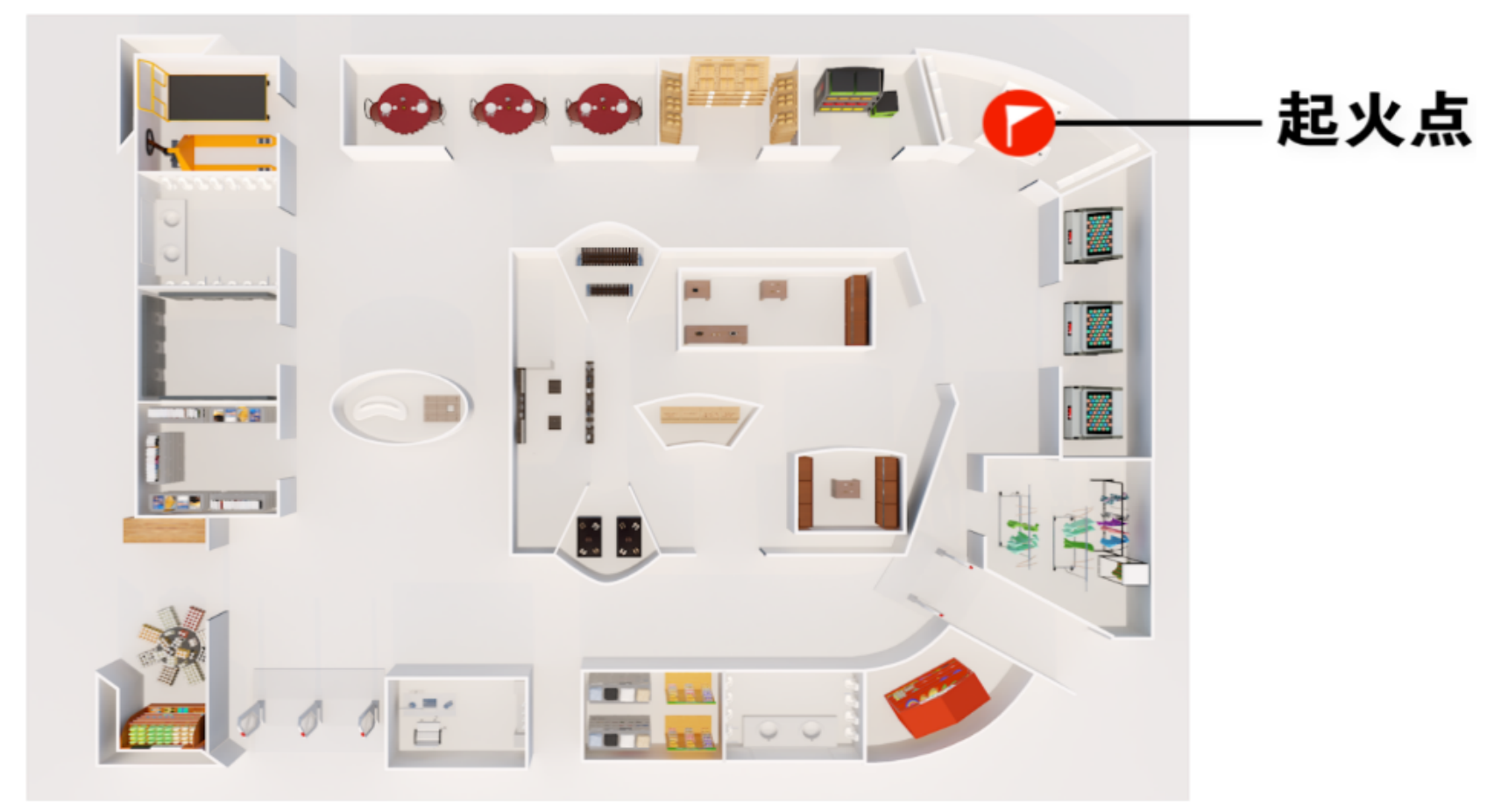

\section{Figure 1}

Scene diagram 


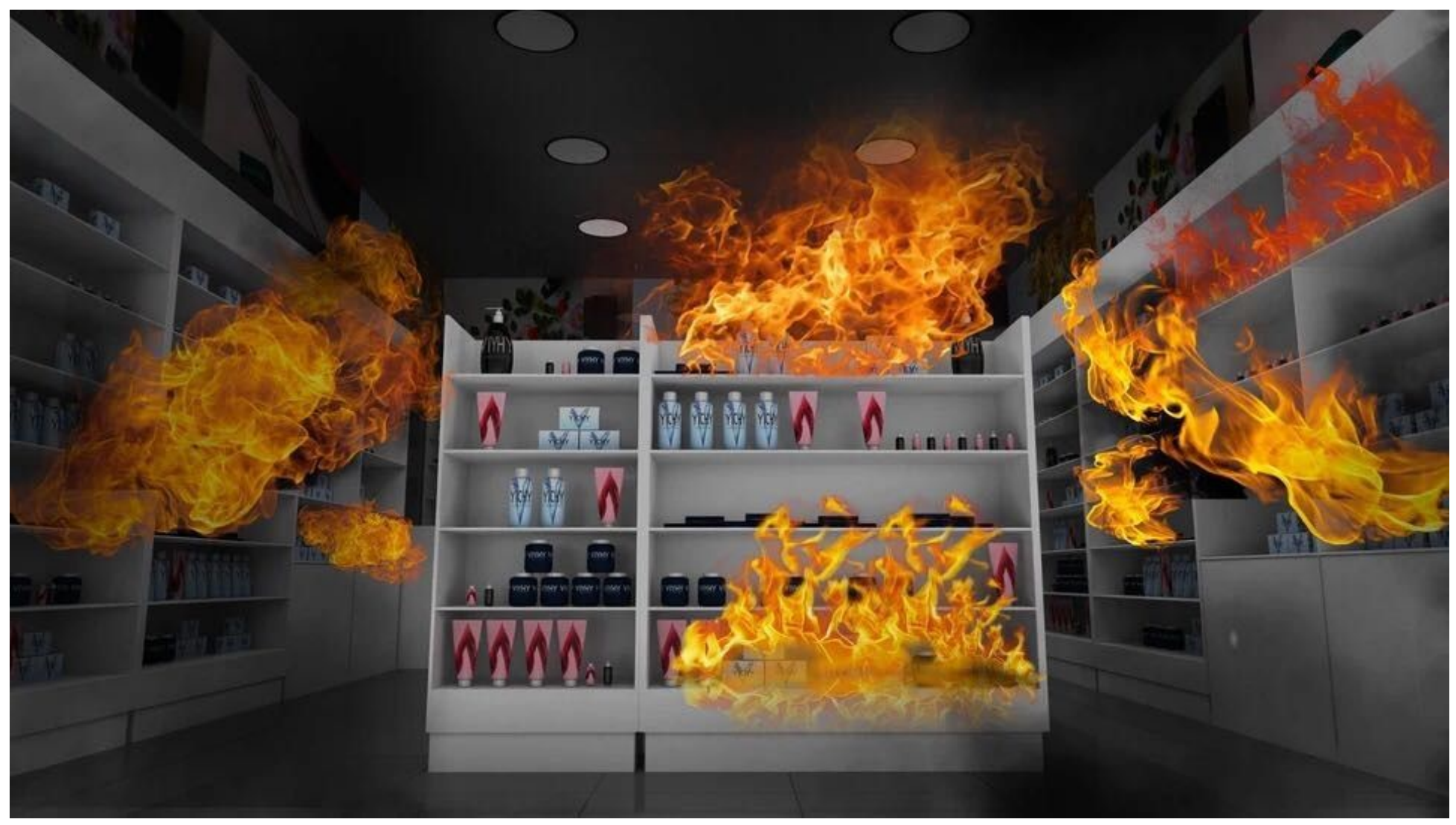

Figure 2

Fire and smoke in the experiment

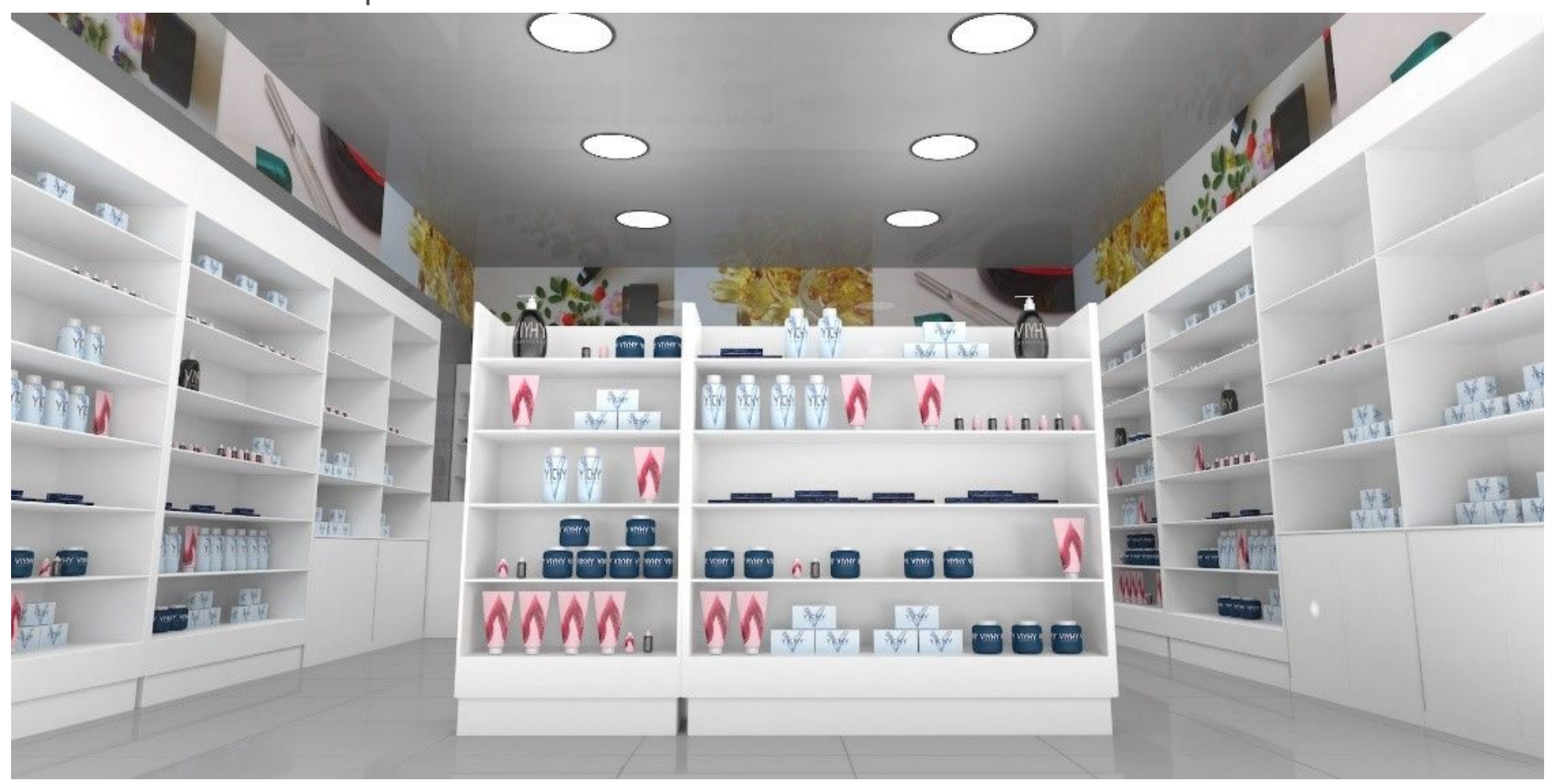

Figure 3 
Promotional spots

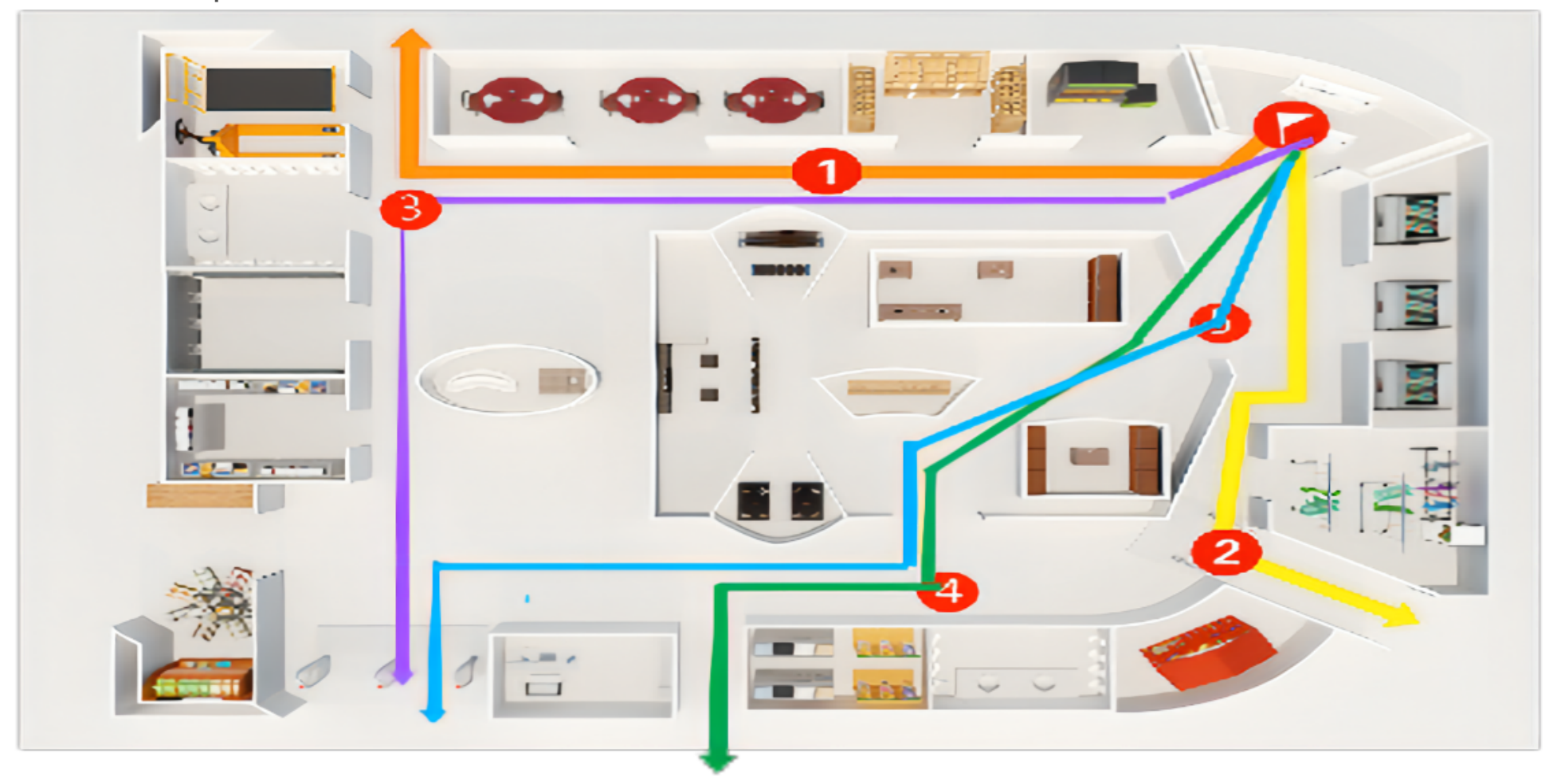

\section{Figure 4}

Evacuation routes

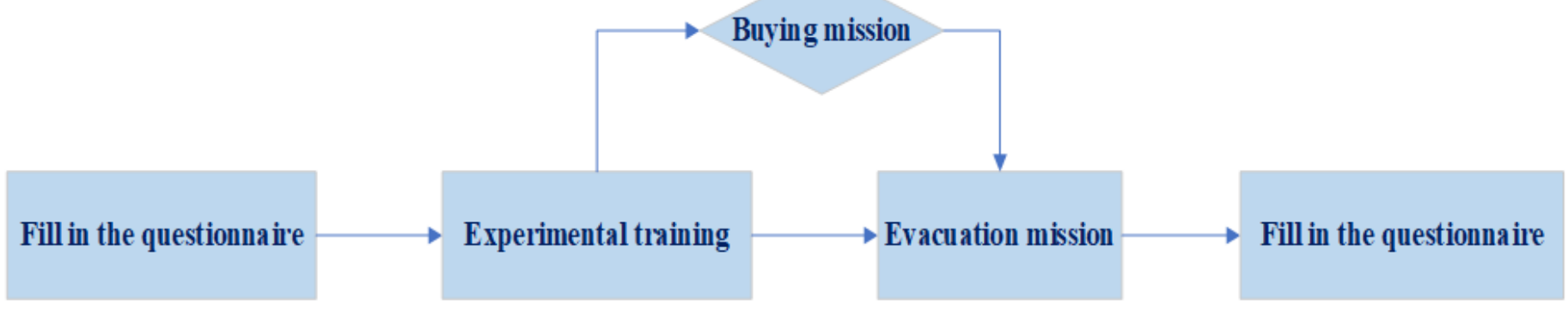

Figure 5

Experimental Procedures 


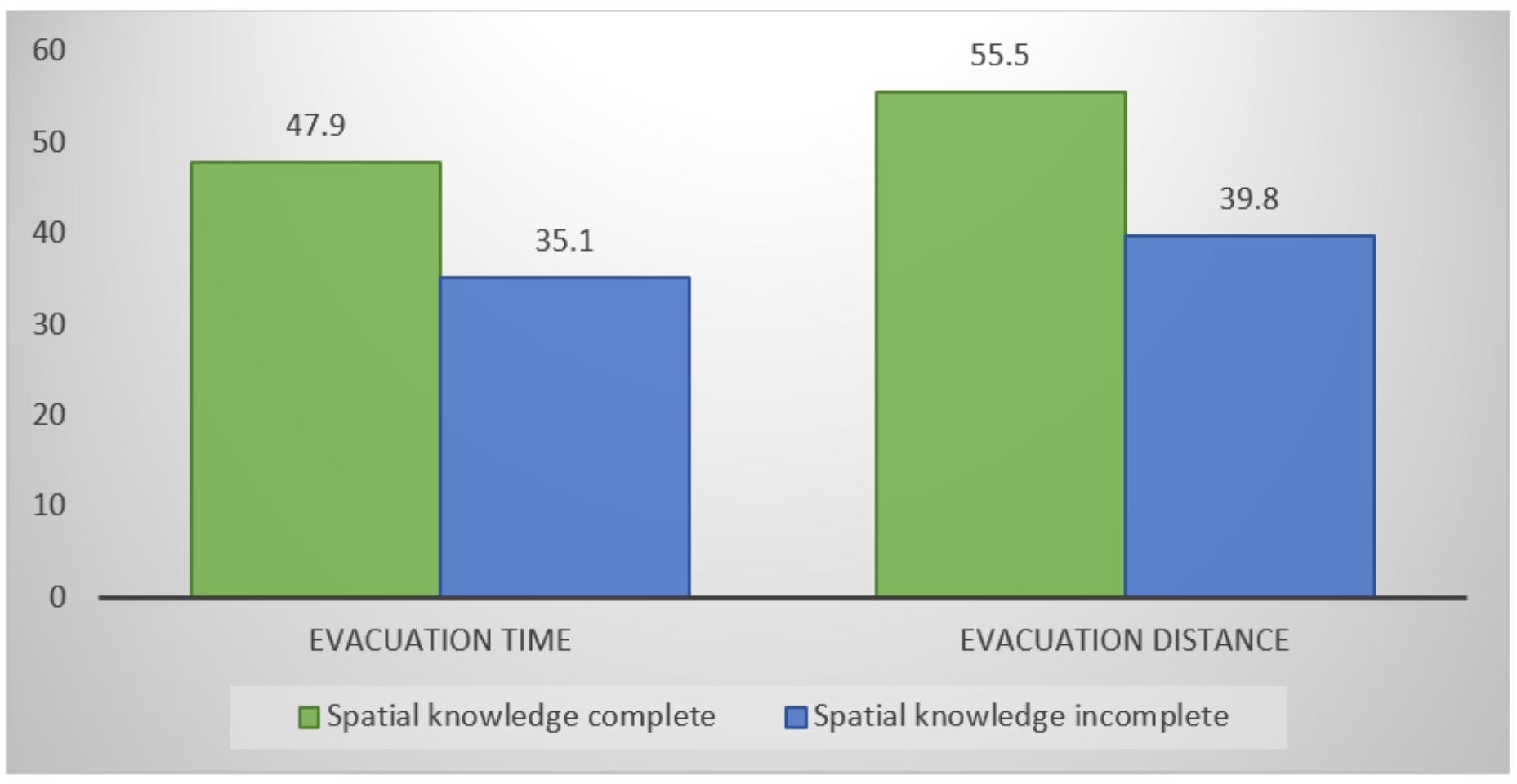

\section{Figure 6}

Influence of spatial knowledge integrity on evacuation

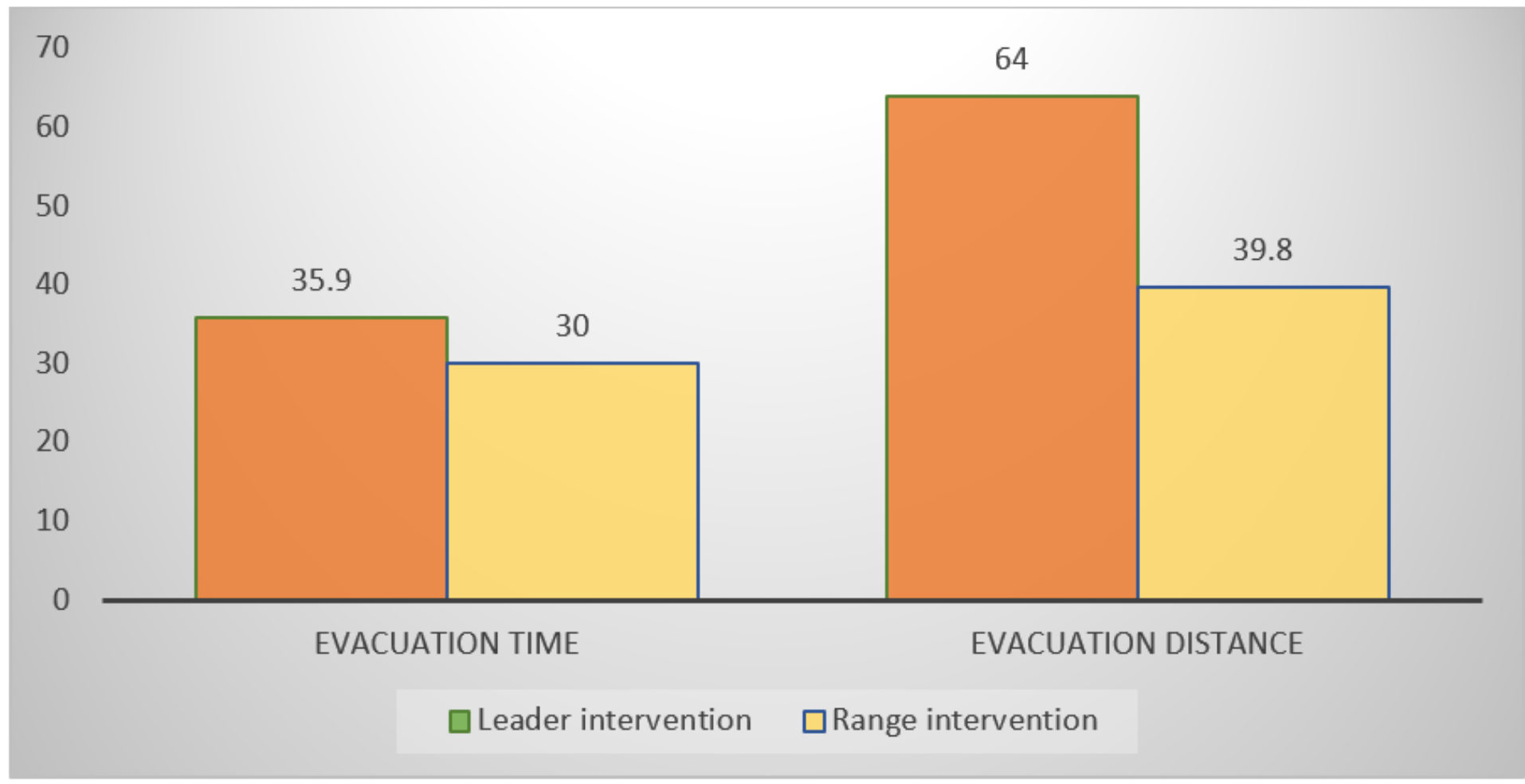

\section{Figure 7}

Influence of different intervention behavior on evacuation 


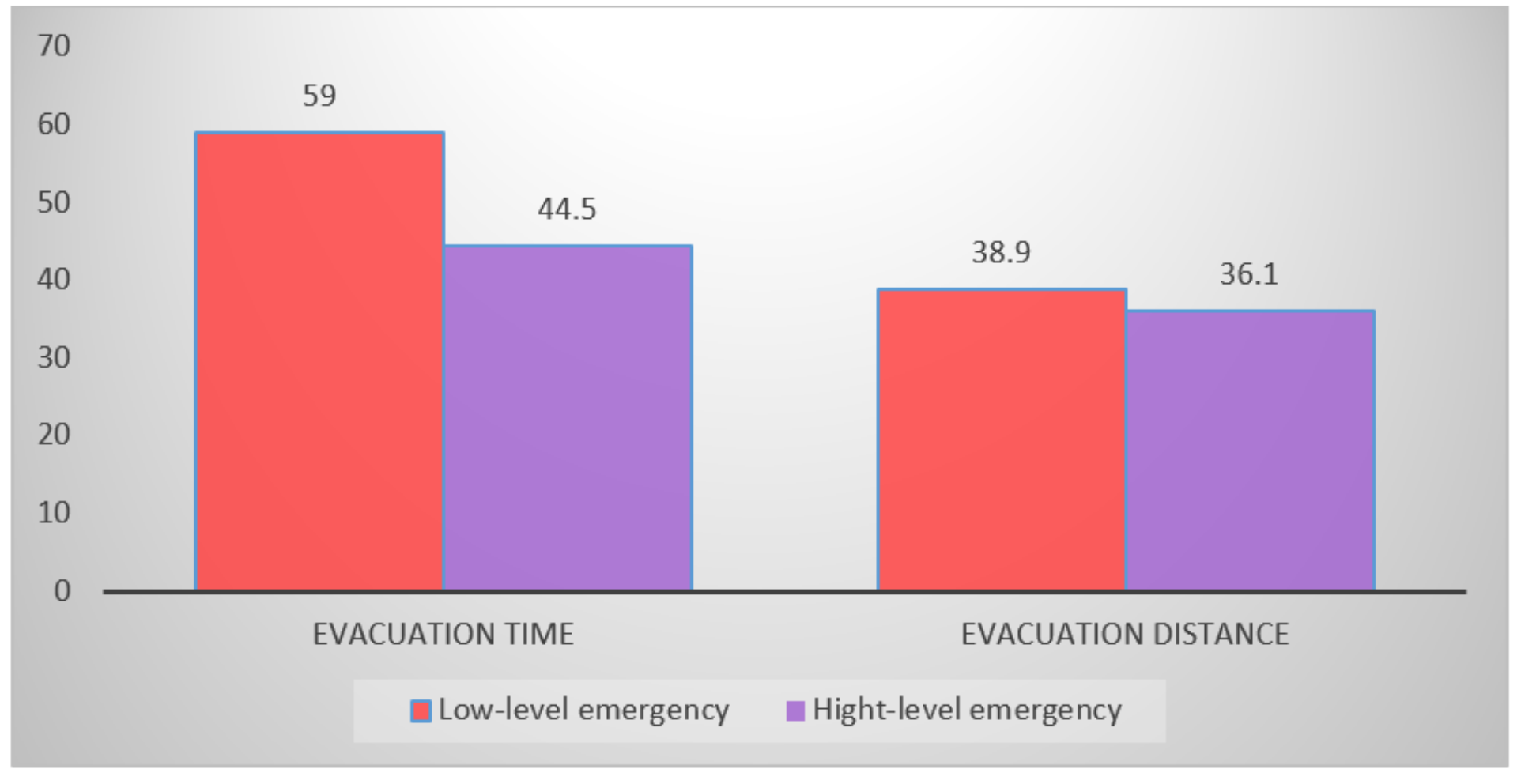

\section{Figure 8}

the impact of different emergency situations 